\title{
TRABAJOS Y LIBROS SOBRE HISTORIA NATURAL Y MEDICINA DE MARTÍN SARMIENTO
}

\section{ROCÍO AMENEIROS RODRÍGUEZ UNIVERSIDADE DA CORUÑA}

\begin{abstract}
RESUMEN: En este trabajo se analizan los escritos de Sarmiento dedicados a la Historia Natural y la Medicina, así como la importante colección de libros que poseía sobre estas materias en su biblioteca. Para ello, se parte de un texto autobiobibliográfico, el Catalogo de los pliègos, y del catálogo de su biblioteca, realizado por el propio autor. Estos documentos permiten estudiar las obras elaboradas por el benedictino y las fuentes bibliográficas que le sirvieron de apoyo, lo que posibilitará describir la aportación y los postulados de Sarmiento sobre estas ciencias y su aplicabilidad, característica esencial de la ciencia ilustrada.
\end{abstract}

PALABRAS CLAVE: Martín Sarmiento, Historia Natural, Medicina, Ciencia, Ilustración española.

\section{WORKS AND BOOKS ON NATURAL HISTORY AND MEDICINE BY MARTÍN SARMIENTO}

\begin{abstract}
In this paper we analyze the writings of Sarmiento dedicated to Natural History and Medicine, as well as to the important collection of books which he owned on these subjects in his library. This analysis is based on an autobiobibliographic text, the Catalogo de los pliègos, and upon the catalog of his library, which was developed by the author himself. These documents allow us to study the works which were elaborated by the Benedictine and the bibliographical sources that served as a support to him, which will make it possible to describe the contribution and the postulates of Sarmiento on these sciences and their applicability, an essential characteristic of illustrated science.
\end{abstract}

KEYWORDS: Martín Sarmiento, Natural History, Medicine, Science, Spanish enlightement. 


\section{Introducción}

No es preciso presentar la figura de Martín Sarmiento, cuyo papel en el movimiento renovador de la ciencia española en el siglo XVIII es bien conocido. Escribe obras e informes a lo largo de su vida que, aunque inéditos por su negativa a publicar para evitar polémicas, fueron conocidos entre las élites de la España del momento. Tuvo muy variados intereses culturales, pedagógicos, bibliográficos, etc., pero especialmente filológicos y de Historia Natural, y siempre con la vista puesta en el desarrollo social y cultural de España. Reunió en su celda del monasterio de San Martín de Madrid una importante y voluminosa biblioteca que le serviría de apoyo para sus obras.

El interés de Sarmiento por la Historia Natural queda patente en los trabajos que dedicó a este campo, en los que suelen ir de la mano la Historia, la Lingüística y la Pedagogía en lo que atañe a los métodos de aprendizaje e investigación. Igualmente, esta afición está presente también en sus postulados acerca de la Medicina y en otras de sus actividades. Recordemos que Sarmiento reunía y clasificaba material botánico y que realizó diversas propuestas en este campo, como la redacción de un Cathalogo de todas las voces vulgares de la Historia natural en toda su latitud ${ }^{1}$. En 1764, en una carta a Andrés Pedro Du-Pont, no duda en justificar este interés en la Regla de San Benito, ni en citar cómo Casiodoro aconsejaba a sus monjes la Historia Natural, "Discite naturas Herbarum", aunque él mismo en dicha carta confiesa modestamente tener una "no aversión" a la Historia Natural lamentando que, al no participar en viajes científicos, a excepción de sus dos visitas a Galicia, no pueda avanzar en este estudio ${ }^{2}$.

El objeto de este trabajo es presentar los textos médicos y de Historia Natural escritos por Sarmiento y la valiosa colección de libros que poseía sobre estas materias, ya que nuestro autor siempre combinó el trabajo empírico con la consulta de su rica biblioteca. Para ello, nos basaremos en un texto autobiobibliográfico, el Catalogo de los pliègos ${ }^{3}$ (cuya redacción

\footnotetext{
${ }^{1}$ SARMIENTO, Martín: Carta en respuesta al Rmo. Pe. Mro. Esteban de Terreros, sobre el origen de la lengua gallega y sobre la paleographia española, AGFCMS, CM, T.IV, 1755, fol. 357v.

${ }^{2}$ SARMIENTO, Martín: "Carta sobre la historia natural, escrita á un caballero, residente en Londres, (1764)", Correo Literario de la Europa, 56, 1782, p. 411.

3 SARMIENTO, Martín: Catalogo de los pliègos, que yo Fray Martin Sarmiento, Benedictino: y professo en $S^{n}$ Martin de Madrid; he escrito, de mi mano, pluma, y letra, sobre diferentes asuntos, BNE, 1767, Ms. 17642. El Catalogo de los pliègos fue publicado en varias ocasiones en base a una copia manuscrita realizada por un contemporáneo de Sarmiento, el también benedictino José Goyanes. Esta copia, que se conservaba en la Biblioteca Provincial de Ourense hasta que fue destruida en un incendio en 1927, nos lleva a pensar que, más que admitir la existencia de una segunda redacción ampliada original de Sarmiento, tal vez perdida y reproducida por Goyanes, estamos ante un documento en el que el propio Goyanes amplió y precisó sus noticias, algunas leídas en los textos de Sarmiento, otras, al parecer, de las múltiples conversaciones que mantuvo con él, adaptándolas incluso al nuevo contexto.
} 
finaliza en 1767, pocos años antes de su fallecimiento) y en el catálogo de su biblioteca ${ }^{4}$ (que redacta desde la década de los años 40 hasta sus últimos días).

El primero de estos escritos es un texto que se enmarca en la larga tradición de confluencia entre el género bibliográfico y el biográfico, y dentro de este último en las autobiografías, que en el análisis de Durán López ${ }^{6}$ se sitúan en la transición entre las antiguas vidas literarias y las modernas biografías. Si exceptuamos las breves líneas referidas a su infancia, el Catalogo de los pliègos constituye un relato sobre su vida intelectual y el contexto en el que se desarrolla, y nos deja ver su continua dedicación a las ciencias, cuestión que estudiamos también a través de sus escritos y analizaremos en este trabajo.

El catálogo de su biblioteca nos permite conocer buena parte de sus lecturas, aunque Sarmiento utilizó también otras bibliotecas y libros prestados. Ordenado por materias, el catálogo sigue, en general, el orden alfabético de estas ${ }^{7}$. Como una división más, entre los libros de Historia coloca Sarmiento los numerosísimos fondos de la Historia Natural (212 títulos en 301 volúmenes), bajo el título Historia Natural, Botánica, y Agricultura. La Historia Natural abarcaba, como señala Sarmiento, la Botánica, la Zoología y la Geología, y se sustentaba, al igual que la Bibliografía, en la descripción, clasificación y listado de las "cosas naturales" . Para Sarmiento la Historia Natural era el fundamento de todas las ciencias, como le indica al duque de Medinasidonia: "Que la Historia Natural, en toda su amplitud, sea el

Entre las publicaciones que se hicieron de esta copia se puede destacar la de Juan Domínguez Fontela, Fray Martin Sarmiento. Su autobiografía (1924), y la de Luis Viñas Cortegoso (1952), con el mismo título de la copia Vida y viajes literarios: número y calidad de los escritos del Rvdmo. P. M. Fray Sarmiento... pero, en este caso, cotejada con el autógrafo de la Biblioteca Nacional, comparando y separando los añadidos de Goyanes y el original de Sarmiento. El texto de Domínguez Fontela se publicó en dos ocasiones más (1972 y 1994). Asimismo, Santos Puerto elaboró un trabajo, en el que presenta una biobibliografía actualizada sobre Sarmiento, reproduciendo el original de la Biblioteca Nacional y aclarando en las notas muchos de los apuntes dados por Goyanes: Una bio-bibliografía actualizada de Martín Sarmiento: Catálogo de los pliegos... sobre diferentes asuntos (2002). Y Henrique Monteagudo y Xosé Manuel Soutullo en Sobre a lingua galega (2002) incorporan una traducción de este texto al gallego, siguiendo la edición de Viñas Cortegoso. Sobre el documento original del Catalogo de los pliègos conocemos un nuevo trabajo, Ameneiros Rodríguez, Rocío: "Vida literaria de Martín Sarmiento: orden y redacción bibliográfica”, Estudios Mindonienses, 34, 2020-2021 (en prensa).

4 SARMIENTO, Martín: Catalogo de los Autores, de quienes, yo Fr. Martin Sarmiento, Benedictino tengo ad usum, ò todas sus obras, ò parte de ellas, ó algun tomo suelto, y separado, RAH, 174?-176?, Ms. 9/1829.

${ }^{5}$ Sarmiento inició el índice de autores del catálogo de su biblioteca en la década de los 40, tal y como figura en el propio manuscrito, Hoy [blanco] de [blanco] de 174. Y mantendrá el registro de las obras y de los autores hasta sus últimos días, ya que incluye obras publicadas pocos años antes de su fallecimiento, como es el caso del Catalogo de su Gabinete de Historia Natural, de Pedro Franco Dávila (París, 1767), y el Viage de M.r Viron, al Sur, traducido por Casimiro Gómez de Ortega (Madrid, 1769).

${ }^{6}$ DURÁN LÓPEZ, Fernando: Vidas de sabios: el nacimiento de la autobiografía moderna en España (17331848), Madrid, Instituto de la Lengua Española, 2005.

${ }^{7}$ Para un análisis más detallado, Varela Orol, Concha y Ameneiros Rodríguez, Rocío: "La biblioteca de Martín Sarmiento. Distinguir para unir", Anales de Documentación, 19 (1), 2016, pp. 1-15, [en línea], doi: http://dx.doi.org/10.6018/analesdoc.19.1.242061.

${ }^{8}$ VALERO, José Antonio: "Una disciplina frustrada: la historia literaria dieciochesca", Hispanic Review, 64 (2), 1996, p. 172. 
primitivo y principal fundamento de toda ciencia humana es para mi una verdad notoria..."9, lo que le permite afirmar a Colmeiro ${ }^{10}$ que nuestro autor fue el español de su tiempo más entusiasta de la Historia Natural.

También en su correspondiente lugar alfabético se encuentra en el catálogo de su biblioteca la Medicina. Cirugía y Química, con una colección algo menor (137 títulos en 177 volúmenes). Igualmente, en este campo, Sarmiento propondrá la necesidad de la aplicación del método científico, atribuyéndosele la redacción del artículo 50 de los Estatutos de la Real Academia de Medicina: "El fin primero e idea general de la Academia será manifestar las verdades y provechosas máximas de la Medicina y Cirugía por el camino de la observación y la experiencia""11.

\section{Observando y escribiendo}

En 1726 Sarmiento escribe, como él mismo denomina, una "Carta-Apologetica", un texto titulado Martinus contra Martinum ${ }^{12}$, con el que inicia la defensa de los planteamientos de Feijoo y que reiterará, años más tarde, en su Demonstracion critico-apologetica de el Theatro Critico Universal (1732). En esta carta, Sarmiento, en un tono sumamente crítico, defiende el primer tomo del Theatro Critico Universal de Feijoo (1726), concretamente el Discurso V dedicado a la Medicina. Esta defensa la realiza como respuesta al texto de Juan de Lesaca, que hace duros ataques a las opiniones de Feijoo acerca de la Medicina y la práctica médica.

Antes de describir esta polémica, queremos detenernos en el hecho de que Sarmiento hace una respuesta manuscrita a un papel manuscrito, lo que nos sitúa ante la perspectiva de un debate científico bajo un medio que sigue sirviendo a los intereses de la comunicación cuando ya la imprenta gozaba de una dilatada historia. En este sentido, no hay que minimizar la influencia de la obra sarmentina, pues si bien en su mayor parte no será editada durante la vida del benedictino, ello en ningún caso significa que no fuese conocida e influyente. Como han demostrado los trabajos de Miguel Benítez ${ }^{13}$, para los textos prohibidos, y de Fernando

\footnotetext{
${ }^{9}$ SARMIENTO, Martín: Cartas al Duque de Medinasidonia, 1747-1770, José Santos Puerto (ed.), Ponferrada, Instituto de Estudios Bercianos, 1995, p. 190.

${ }^{10}$ COLMEIRO, Miguel: La Botánica y los botánicos en la Península Hispano-Lusitana. Estudios biográficos y bibliográficos, Madrid, Impr. de M. Rivadeneyra, 1858, p. 165.

11 FILGUEIRA VALVERDE, José: "Para las ideas médicas del Padre Sarmiento (1695-1772). Los Apuntamientos inéditos”, en Libro de los amigos de José Luis Barros, A Coruña, Edicións do Castro, 1990, p. 94.

12 SARMIENTO, Martín: Martinus contra Martinum, AGFCMS, CM, T.I, 1726, fols. 95r.-229r.

${ }_{13}^{3}$ BENÍTEZ, Miguel: La face cachée des lumières: recherches sur les manuscrits philosophiques clandestins de lâge classique, París, Universitas, 1996.
} 
Bouza $^{14}$, para el género de educación de príncipes, el manuscrito como soporte de la información tendrá todavía larga vida después del nacimiento del libro impreso.

La respuesta de Sarmiento a Lesaca ha de contextualizarse en un tipo de polémicas en torno a la Medicina, en las que ya desde hacía años Lesaca había atacado los escritos de los novatores Martín Martínez, Diego Mateo Zapata y Alejandro Avendaño, quienes también contestaron a Lesaca. Estas disputas entre la filosofía natural aristotélica y la nueva filosofía experimental eran públicas en los cenáculos cortesanos y de interés para los pacientes de ambos tipos de remedios terapéuticos ${ }^{15}$.

Sarmiento, en su defensa de Feijoo, prima la observación y la experiencia: los datos empíricos proporcionados por la práctica clínica deben constituir el principio del arte de curar. Asimismo, defiende la necesidad del conocimiento de la Química, que debía considerarse como uno de los fundamentos de la naturaleza, y cree y apoya firmemente la fuerza sanadora de esta, una corriente que en su vertiente terapéutica abogará por la utilización de remedios naturales, recogiendo la tradición médica hipocrática ${ }^{16}$. Así, en Martinus contra Martinum, nuestro autor conjuga las posiciones del movimiento de los novatores, que cuestionan por primera vez el paradigma galénico desde los presupuestos de la llamada iatroquímica, con la medicina hipocrática, que será su gran apuesta terapéutica. Las virtudes medicinales de las plantas, de determinados animales y productos exóticos, así como las aportaciones de la medicina popular, constituirán su alternativa a la medicina galénica. En este sentido, Sarmiento expresará a lo largo de toda su obra un rechazo hacia los profesionales de la medicina de su época y las prácticas de las sangrías y las purgas a las que eran tan aficionados, y se declarará defensor del uso terapéutico de la "carqueixa" (carquesa, carqueja en español), que llega a circular por México gracias a sus envíos ${ }^{17}$.

En la década de los años 30 nuestro autor comienza a recibir encargos de trabajos por parte de instituciones civiles y de gobierno, tales como la solicitud de realizar emblemas para la Real Academia Nacional de Medicina y la Real Congregación de Hospitales. Sarmiento

\footnotetext{
14 BOUZA, Fernando: Corre manuscrito: una historia cultural del Siglo de Oro, Madrid, Marcial Pons, 2001.

15 PARDO TOMÁS, José: "La medicina en España a finales del siglo XVII: Diego Mateo Zapata”, en La ciencia europea desde 1650 hasta 1800: actas XIII y XIV del Seminario Orotava, La Orotava, Fundación Canaria Orotava de Historia de la Ciencia, 2007, [en línea], http://hdl.handle.net/10261/44335.

16 GARCÍA GUERRA, Delfín: "La medicina en la obra del Padre Sarmiento", en O Padre Sarmiento e o seu tempo: actas do Congreso Internacional do Tricentenario de Fr. Martín Sarmiento (1965-1995), Santiago de Compostela, Consello da Cultura Galega, Universidade, 1997, T.II, pp. 348-352.

${ }^{17}$ En 1759 Sarmiento escribe De la planta Carqueixa (AGFCMS, CM, T.XI, fols. 323r.-336r.), un trabajo que sería editado en 1786, 1787 y 1790 con el título Disertacion sobre las virtudes maravillosas y uso de la planta llamada carqueixa. Y en 1761 nuestro autor realiza Discurso Sobre El Nombre; Genero; Analysis y Virtudes de la Planta... Carqueixa (AGFCMS, CM, T.XI, fols. 338r.-411r.), que aparece citado en el listado de los escritos de las décadas de los 50 y 60 del Catálogo de los pliègos (Véase p. 9).
} 
sugiere un cuerpo y un alma para cada una de ellas: el cuerpo es la imagen y el alma es el mote, texto o verso que le rode ${ }^{18}$. El actual emblema de la Academia de Medicina es una de esas propuestas hechas por nuestro benedictino: "Pintese un sol arrojando sus rayos Paralelos à un Espejo Ustorio Parabolico, y pongase un Pedazo de oro en el foco, como que se derrite. Mote, Et major Collectis Viribus exit" ${ }^{\prime 1}$.

En 1745 Sarmiento realiza su primer viaje a Galicia, en el que va anotando sus observaciones sobre el mundo natural y que acabará completando con los libros de su celda, como indica en los Pensamientos critico-botanicos y en la carta escrita a Andrés Pedro Du-Pont:

\begin{abstract}
"Me dediqué à repasar mis Quadernos de vegetables y de Historia Natural, y à rectificar por los Libros, todo lo que avia observado en mis dos Peregrinaciones à Galicia en 745 y 754”20.

"Con esta ocasión viendome sin libros, quise que los Mixtos naturales me sirviesen de libros vivos y originales; ya que recluso en mi celda me habian servido de Mixtos pintados y muertos los que tengo en mis libros de Historia Natural.

Muy raro será el animal, pez, ave, crustaceo, testaceo, insecto, vegetable, fosil, petrificacion, metal, etc. cuya singular pintura, ó exâcta descripción no tenga yo en mis libros, antiguos de la edad media, y modernos" 21 .
\end{abstract}

En 1750, Sarmiento escribe a Francisco Rávago ${ }^{22}$ sobre el cerco de las sardinas restablecido en 1749, en la ría de Pontevedra, por su hermano Francisco Javier, ministro de marina. No nos cabe duda de que el informador in situ de Sarmiento era Francisco Javier, a quien nuestro benedictino proporcionará, a lo largo del tiempo, recomendaciones sobre variados asuntos de Historia Natural (la calidad de la tierra, las plantas y sus propiedades, los peces, los minerales, los moluscos...). Prueba de ello son las numerosas cartas que nuestro autor redacta para su hermano entre 1757 y $1760^{23}$.

En 1752 Sarmiento escribe para el conde de Maceda, Antonio Pedro Nolasco de Lanzós y Taboada, un trabajo Sobre el Animal Zebra ${ }^{24}$, uno más de los que muestran su afición

${ }^{18}$ Dichas propuestas se encuentran en la Colección Medina Sidonia con los títulos: Apuntamientos para formar una Empresa, según todo el rigor de las leyes, que pueda servir para la nueva Academia Medico-Matritense (AGFCMS, CM, T.V, 1734, fols. 48r.-72v.); y Pídese una Empresa, Emblema o Blasòn, Insignia, Armas, ò distintivo para la nueva R! Hermandad, ò Congregacion, que se quiere establecer, para la mejor asistencia de los Enfermos en los Hospitales (AGFCMS, CM, T.V, 1750, fols. 74r.-82r.).

${ }^{19}$ SARMIENTO, Martín: Apuntamientos para formar una Empresa..., fol. 51r.

${ }^{20}$ SARMIENTO, Martín: Pensamientos critico-botanicos para emprehender, $y$ conseguir, en pocos años una bistoria general completa, de todos los vegetables que nacen en España, AGFCMS, CM, T.X, 1754-1762, fol. 373r.

${ }^{21}$ SARMIENTO, Martín: "Carta sobre la historia natural, escrita á un caballero, residente en Londres, (1764)", p. 411.

22 SARMIENTO, Martín: Sobre el cerco de Pontevedra, AGFCMS, CM, T.IX, 1750, fols. 1r.-32r.

${ }^{23}$ SARMIENTO, Martín: Fray Martín Sarmiento. Epistolario, José Filgueira Valverde y María Xesús Fortes Alén, (eds.), Santiago de Compostela, Consello da Cultura Galega, 1995.

${ }^{24}$ SARMIENTO, Martín: Sobre el Animal Zebra, que se criaba en España, MP, CM, T.XII, 1761, fols. 172r.-216v. 
por la Historia Natural. En este texto nuestro autor, a partir de diversas fuentes documentales, considera que la cebra o un animal muy parecido se conocía y apreciaba en España en la época medieval y que ese animal, extinguido ya, era muy semejante al que vive en África, proponiendo su restitución en nuestro país. Sarmiento insistió en esta idea, así en 1760 y 1770, en respectivas cartas dirigidas al duque de Medina Sidonia, vuelve a plantear esta cuestión $^{25}$ y también en la Obra de 660 pliegos, 1762-1766 26.

En 1754 Sarmiento realiza el conocido como el segundo de sus viajes a Galicia. En dicho viaje, como en el de 1745, elabora un diario en el que anota sus jornadas ${ }^{27}$. En este momento, Sarmiento intensifica su dedicación a la Historia Natural y señala los métodos de investigación por los que adquiría el conocimiento, basados tanto en el trabajo de campo como en el de gabinete. De este trabajo dedicado a la Historia Natural, y en especial a la Botánica, surgen diferentes textos, los cuales se reúnen en la copia de Medina Sidonia bajo el título Apuntamientos para una botanica española ${ }^{28}$.

En el Catalogo de voces de diferentes vegetables (1754-1758 ${ }^{29}$, nuestro autor copia en primer lugar las voces de las plantas recogidas en su diario del viaje de 1745 y en los primeros cuadernos que componen el Cathalogo de vozes y frases de la lengua gallega (1745-1746 y 1754$1755)^{30}$. Suponemos que esta primera parte, formada por un listado de nombres, la copia antes de salir al viaje de 1754 para reunir todas las voces de las plantas en un mismo documento, tanto las recogidas desde el viaje anterior, como las que pudiese apuntar a partir de este. En definitiva, en el Catalogo de voces de diferentes vegetables se recogen de forma numerada los nombres vulgares de las plantas que Sarmiento anotó en los viajes que hizo a Galicia en 1745 y 1754, y en su trabajo de gabinete en Madrid, acompañados además de algunas pequeñas ilustraciones y de las citas de las fuentes que maneja.

El Onomàstico latin-gallego de los vegetables. Systèma de Mr. Tournefort $(1754-1755)^{31}$ es un texto en el que nuestro benedictino clasifica las plantas con el sistema taxonómico de Tournefort, remitiendo a la página correspondiente de la obra Institutiones rei herbariae del botánico francés.

\footnotetext{
25 SARMIENTO, Martín: Cartas al Duque de Medinasidonia, 1747-1770, pp. 176-178 y pp. 233-235.

${ }^{26}$ SARMIENTO, Martín: Obra de 660 pliegos: De historia natural y de todo género de erudición, Henrique Monteagudo (ed.), Santiago de Compostela, Consello da Cultura Galega; Madrid, CSIC, Vol. II, 2008, pp. 29-30.

${ }_{27}$ SARMIENTO, Martín: Viage que el Padre Sarmiento biz̧o à Galicia el año de 1754, AGFCMS, CM, T.I, 1754-1755, fols. $553 \mathrm{r} .-675 \mathrm{v}$.

28 SARMIENTO, Martín: Apuntamientos para una botanica española, AGFCMS, CM, T.X, 1751-1758, fols. 1r.-211r.

${ }^{29}$ SARMIENTO, Martín: Catalogo de voces vulgares y en especial de voces gallegas de diferentes vegetables, AGFCMS, CM, T.X, 1754-1758, fols. 27r.-198r.

30 SARMIENTO, Martín: Cathalogo de vozes y frases de la lengua gallega, AGFCMS, CM, T.IV, 1745-1746 y 17541755, fols. 83r.-333r.

${ }^{31}$ SARMIENTO, Martín: Onomàstico latin-gallego de los vegetables. Systèma de Mr. Tournefort, AGFCMS, CM, T.X, 1754-1755, fols. 243r.-297v.
} 
En los Pensamientos critico-botanicos (1754-1762) $)^{32}$, nuestro autor muestra su interés no solo por las voces que designan las plantas y su etimología, sino también por sus utilidades, lo que apoya los ya comentados postulados de nuestro autor sobre la Medicina, en los que defiende el uso de los remedios naturales aprovechando las virtudes medicinales de las plantas y el conocimiento popular. Este texto, como otros escritos del benedictino ${ }^{33}$, deja constancia de los materiales botánicos que recogía en sus viajes y los que recibía en su celda.

En 1756 Sarmiento escribe Sobre los Vegetables Kali, Sosa y Barrilla ${ }^{34}$ a petición del oficial mayor del Despacho Universal de Marina, Juan Antonio Arias, en donde presenta los distintos nombres, orígenes y variedades de estas plantas, especificando las propias de Galicia. Nuestro autor dedica la mitad de este trabajo al método para escribir la Historia Natural y la Botánica, insistiendo en el estudio de campo de todo lo que "nace" en España, su etimología y sus propiedades. Así lo hará él en este mismo texto para la riqueza de Galicia, principalmente vegetal, apoyándose también en diferentes autores, tales como Plinio, Quer, Tournefort, Nicolás Monardes y José de Acosta.

En el Onomastico etymologico de la lengua gallega $(1757-1769)^{35}$ nuestro benedictino trata la importancia del conocimiento de la Historia Natural para el estudio de la etimología, del léxico gallego, de la Escritura y, en general, para el proceso de aprendizaje de cualquier facultad, dejando patente una vez más la relación de la Historia Natural con la Historia, la Lingüística y la Pedagogía.

En las décadas de 1750 y 1760 Sarmiento redacta otros numerosos y variados escritos de Historia Natural $^{36}$, de los que deja constancia, a modo de listado e intercalados entre otros títulos, también en el Catálogo de los pliègos.

Sobre el arbol Azederac (1756)

Sobre plantas de Coria (1757)

Sobre el Rhinoceronte (1762)

Sobre la Betula, o Bidueyro (1759)

Sobre Plantas, de Toledo (1761)

\footnotetext{
32 SARMIENTO, Martín: Pensamientos critico-botanicos..., fols. 299r.-379v.

33 Además de los citados a lo largo de este trabajo, Respuesta sobre unas plantas remitidas de Asturias a Madrid en 1757 (AGFCMS, CM, T.XI, fols. 179r.-183v.).

${ }^{34}$ SARMIENTO, Martín: Sobre los Vegetables Kali, Sosa y Barrilla, AGFCMS, CM, T.XI, 1756, fols. 29r.-146r.

35 SARMIENTO, Martín: Onomastico etymològico de la lengua gallega, AGFCMS, CM, T.III, 1757-1769, fols. 449r.743 r.

${ }^{36}$ La copia de estos trabajos se puede consultar en los tomos XI (AGFCMS ) y XII (MP) de la Colección Medina Sidonia, a excepción de Sobre el Origen de las Bubas: T.V (AGFCMS); Sobre el Lobo Cerval, cuya copia se encuentra en el Archivo del Monasterio de Santo Domingo de Silos (1760, Ms. 72 y Ms. 73/B); y Sobre la Tyraña de Liebana, un texto del que se desconoce su localización (la única referencia al respecto es la mención que del mismo hace Sarmiento en una carta a su hermano Francisco Javier, en Madrid, el 23 de enero de 1760, SARMIENTO, Martín: Fray Martin Sarmiento. Epistolario, pp. 189-191).
} 
Sobre el Lobo Cerval (1760)

Sobre la Bardana, o Lampazo (1760)

Sobre el Papion, o Cynocephalo (1760)

Sobre las Serofularias (1760)

Sobre el Arbol Santo de Aranjuez.(1760)

Sobre la Manzanilla fina (1761)

Sobre el Tillo (1761)

Sobre los Pezes Cetaceos Orcas (1760)

Sobre la Carquéxa, para Mexico (1761)

Sobre la Tyraña de Liebana (1760)

Sobre el Mostajo (1760)

Sobre la Coscara, o Geum, de Tournefort (1760)

Sobre el Lino Aloes, Calice (1762)

Sobre el Origen de las Bubas (1761)

Sobre Las Pizarras Arborizadas de Mondonedo (1751)

Sobre el Phenicoptero o Flamenco, Ave (1757)

Sobre Los Atunesy Almadràvas (1757)

En 1762 Sarmiento redacta un amplio trabajo sobre la planta "seixebra" (teucrio en español): Papel Curioso Sobre la Planta Seyxêbra ${ }^{37}$. Destaca de esta sus múltiples propiedades y su abundancia en Galicia, a la que quiere hacer una contribución con este escrito, especialmente a Pontevedra. Para ello, presenta un estudio detallado y documentado de la "seixebra", poniendo de manifiesto además sus reflexiones acerca del método para escribir y conocer la Historia Natural y la Botánica, y su aplicación en la Medicina. Sobre sus usos terapéuticos afirma: "Demuéstrase; Que con la Seyxêbra; y con mas de 40. Vegetables, sus Symbolos, en Naturaleza y en Virtudes, se podra tener una Botica Española-Casera; sin necesitar de Droguistas ni de Boticario" ${ }^{38}$.

Desde 1762 hasta 1766 Sarmiento trabajó en la Obra de 660 pliegos $^{39}$, un escrito de enorme extensión, en el cual intercalará distintos temas, entre los que destacamos también la Historia Natural y la Lingüística, recordemos, íntimamente relacionadas para nuestro autor con la Pedagogía.

\footnotetext{
37 SARMIENTO, Martín: Papel Curioso Sobre la Planta, que en Galicia es muy Comun y Se llama Seyxêbra, AGFCMS, CM, T.XI, 1762, fols. 422r.-711v.

${ }^{38}$ Ibidem, fols. $429 \mathrm{r} .-429 \mathrm{v}$.

39 SARMIENTO, Martín: Obra de 660 pliegos, MP, CM, T.XIII-XVII, 1762-1766.
} 
En 1766 Sarmiento escribe De el Mixto Vegetable Carolo Santo ${ }^{40}$, dando respuesta a una petición sobre el origen, el nombre y las virtudes de una raíz traída de América. Nuestro autor determina que el carolo santo es la raíz del mechoacán y que el nombre de carolo santo fue asignado por tratarse de un carolo (como se dice en Galicia), un trozo de la raíz, y santo por sus múltiples propiedades, entre las que destaca la de purgante. Aprovecha el texto nuestro benedictino para abogar también por el uso medicinal de las plantas de nuestro país.

En ese mismo año, Sarmiento redacta un nuevo texto en el que estudia la planta que en Galicia llaman "herba balesta, lestra o lesta" (también lesta, alestas o grama de olor en español): De la Grama oloròsa en Gallego Lesta ${ }^{41}$. Se trata de una planta muy aromática, aprovechada principalmente por esta cualidad para uso doméstico y para adobar el tabaco. En este escrito, nuestro autor pone de manifiesto la dificultad de estudiar la Botánica, insiste en el trabajo previo de campo, critica la coordinación de los nuevos sistemas botánicos y propone cultivar las plantas silvestres. Al igual que había hecho en el texto sobre la "seixebra", en donde mencionaba también a la lesta, nuestro benedictino indica que trajo su semilla a Madrid desde Galicia y que, como otras muchas, se la dio a José Quer para que la sembrase en el Jardín Botánico. Para Quer escribe en 1756, Noticia De algunos Vegetables estraños Que ya estan connaturalizados y se cultivan en España ${ }^{42}$, en donde indica que el director del Real Jardín Botánico de Madrid, "me dixo que le diesse la noticia q yo tenia de los Vegetables extraños trahidos à España" ${ }^{43}$.

También en 1766, Sarmiento escribe Discurso Sobre Singularissima Piedra Negra de la Ara, de $\mathrm{Lugo}^{44}$, un texto en el que nuestro autor, apoyándose en diferentes fuentes documentales, determina que las aras de la Catedral de Lugo son idénticas a la de la Catedral de Cuzco y estaban hechas con la misma piedra que le habían enseñado a él en su celda y que acabaría comprando en una almoneda ese mismo año. Se trata de la obsidiana, una piedra negra traída de América, muy dura y brillante. Era una piedra rara y muy valorada puesto que, aunque se había utilizado para hacer espejos, herramientas y armas cortantes, se perdió el modo de trabajarla y, únicamente, se conservan las de los sepulcros. A esta piedra se le atribuían también virtudes medicinales, pero nuestro benedictino muestra su oposición a la aplicación de los minerales y metales en la Medicina.

\footnotetext{
${ }^{40}$ SARMIENTO, Martín: De el Mixto Vegetable Carolo Santo, AGFCMS, CM, T.XI, 1766, fols. 729r.-737v. ${ }^{41}$ SARMIENTO, Martín: De la Grama oloròsa en Gallego Lesta, AGFCMS, CM, T.XI, 1766, fols. 779r.-797v.

${ }^{42}$ SARMIENTO, Martín: Noticia De algunos Vegetables estraños Que ya estan connaturalizados y se cultivan en España ò en tierra, ò en tiestos, AGFCMS, CM, T.XI, 1756, fols. 161r.-167r.,

${ }^{43}$ Ibidem, fol. 166v.

${ }^{44}$ SARMIENTO, Martín: Discurso Sobre Singularissima Piedra Negra de la Ara, de Lugo, MP, CM, T.XII, 1766, fols. $458 \mathrm{r} .-484 \mathrm{v}$.
} 


\title{
3. Leyendo para describir y clasificar
}

En los Pensamientos critico-botanicos Sarmiento expresa:

“...con el ansia del mayor acierto comprè muchissimos libros de Botanica, y de Historia Natural, que ni antes tenia ni avia visto, y los mas caros, selectisimos, y modernos. De manera, que de 6.500 volumenes, que oy tengo ad usum, a 15 de Mayo de 1762, los 300 son de Botanica e Historia Natural, ademas de los Libros periodicos de Academias, Actas, Colegio curioso, Viageros, etc." 45

En la relación de libros de su biblioteca Sarmiento incluye en la Historial Natural los libros de Agricultura y de Farmacia. La ciencia ilustrada es una ciencia en la búsqueda del bienestar, una ciencia que persigue su aplicabilidad. Es esta perspectiva la que explica la reunión de las tres materias, ya que el desarrollo de la agricultura precisaba de la aplicación de los conocimientos botánicos y la formación en este campo es esencial para los boticarios. Como bien explicaría bastantes años después Georges Cuvier, la Botánica fue siempre más cultivada que la Zoología, "la raison de cette saveur, c'est qu'elle a eté considerée comme étant d'une utilité plus générale" ${ }^{\text {"6 }}$. Sarmiento destaca que la Botánica, a la que llamará “divertidísima ciencia”, esté de moda en los países extranjeros y pese a eso se ignore en España. Más aún, respecto a la producción española sobre Historia Natural, afirma:

\begin{abstract}
"No solo han sido los gallegos omisos a cultivar su idioma, sino que igualmente lo son en observar la Historia Natural de Galicia y en dedicarse a la Botánica; es verdad que esta omision y esta desidia igualmente la tienen los castellanos y otras provincias de España. De casi un millón de libros que hay en las naciones extranjeras sobre la Historia Natural en sus tres reinos: mineral, vegetal y animal, es preciso apurar las librerías para encontrar dos o tres docenas de autores españoles que hayan escrito de estas materias o en español o en latín" ${ }^{47}$.
\end{abstract}

Conjugando los intereses botánicos, filológicos, farmacológicos y agrícolas, sintetiza lo que los novicios deben aprender del mundo de los vegetales:

" $1^{\circ}$ el nombre castellano, y el latino correspondiente. $2^{\circ}$ el uso que tiene en la vida civil. $3^{\circ}$ qué alimento es, y qual es su virtud específica en la Medicina, o Cirugía. $4^{\circ}$ el modo de cultivar y promover ese vegetable. Omito otros conocimientos Physicos, pues no pretendo, sino como en todo lo demas, que el muchacho comience en aquella edad à ser un Botánico de devoción"48.

\footnotetext{
45 SARMIENTO, Martín: Pensamientos critico-botanicos..., fol. 373r.

46 CUVIER, Georges: Histoire des sciences naturelles, París, Chez Fortin, Masson y C., 1841, p. 5

${ }^{47}$ SARMIENTO, Martín: Apuntamientos para un discurso apologetico sobre etymologias, y methodo para aplicar los elementos etymologicos al onomastico de la lengua gallega, AGFCMS, CM, T.IV, 1758, fol. 495v.

48 SARMIENTO, Martín: Notas al Privilegio de Ordoño II, AGFCMS, CM, T.XVIII, 1752, fol. 167r.
} 
La ordenación de esta colección de Historia Natural, Botánica, y Agricultura en el catálogo de la biblioteca de Sarmiento sigue el esquema propuesto por nuestro autor en su Catalogo de libros curiosos y selectos ${ }^{49}$, en donde pensaba que cada ciencia debía abrirse con su historia y progresos. Leer este epígrafe del catálogo supone un repaso de la historia de la ciencia natural desde la antigüedad clásica hasta el siglo XVIII.

En dicho epígrafe ${ }^{50}$ comienza la relación de libros de Historia Natural con cuatro versiones de la obra de Dioscórides, todas del siglo XVI (aunque una de ellas en edición del siglo XVIII), versiones que no carecieron de influencias y conflictos entre ellas. Nos referimos a la versión greco-latina de Antonio Saraceno (Fráncfort, 1598), la del físico portugués Amato Lusitano (Lyon, 1558) y la comentada por Pietro Andrea Mattioli, con adiciones de Bahuin (Fráncfort, 1598). La cuarta versión es la del médico Andrés Laguna, con adiciones del médico salmantino Suárez Ribera, en su primera edición de 1733 (Madrid). Pasados los años, como se aprecia por la letra del catálogo, nuestro autor comprará otra edición del siglo XVI (Lyon, 1554), "un tomito muy manual"51.

La edición de Laguna debía de ser muy valorada por Sarmiento, pues su autor había realizado un considerable trabajo filológico y, además, la información botánica procedía del estudio directo de las plantas por toda Europa ${ }^{52}$, juntándose "las voces" y "las cosas" en el texto, como era del gusto de Sarmiento.

Laguna había tenido en cuenta para la elaboración de su trabajo también la obra de Plinio, otro de los autores clásicos presentes en la biblioteca de Sarmiento con su Historia Natural en la edición latina de 1631 (Colonia), en la edición castellana del médico Gerónimo Huerta (Madrid, 1624) y en la edición de 1741 (Basilea) comentada por Jean Hardouin. No podían faltar las obras de Teofrasto, Sorano de Éfeso, Ateneo de Náucratis, Oribasio, Apuleyo Bárbaro y Antonio de Musa, reunidas en una edición con el título De re medica (Basilea, 1528), de Andreas Cratandri.

Después de los clásicos greco-latinos se incluyen en el catálogo los autores medievales, como Petrus Crescencius, Bartholomeus Anglicus y la Escuela Médica Salernitana, con su Regimen Sanitatis, anotado por Arnau de Vilanova (Róterdam, 1649). El

49 SARMIENTO, Martín: Catalogo de algunos libros curiosos y selectos, para la libreria de algun particular, que desee comprar de tres á quatro mil tomos, AGFCMS, CM, T.VI, 1748, fols. 107r.-181v.

${ }^{50}$ El epígrafe de Historia Natural, Botánica, y Agricultura ha sido transcrito por Pensado, SARMIENTO, Martín: "Escritos menores de Fr. Martín Sarmiento", en José Luis Pensado (ed.), Estudos adicados a Fr. Martín Sarmiento: artigos tirados dos Cuadernos de Estudios Gallegos (1945-1982), Santiago de Compostela, Instituto de Estudios Gallegos «Padre Sarmiento», 1995, pp. 533-545.

51 SARMIENTO, Martín: Catalogo de los Autores, de quienes, yo Fr. Martin Sarmiento..., fol. 142v.

52 GÓMEZ MORENO, Ángel: “La resurrección de Dioscórides y la edición comentada de Laguna”, Criticón, 79, 2000, pp. 107-122. 
que se considera el primer libro impreso de Historia Natural, salido de las imprentas de Schöffer en 1498, está entre estos libros en la versión latina publicada en 1517 (s.l.), el Hortus sanitatis de Johannes de Cuba.

También la ciencia botánica de los siglos XVI y XVII está representada en la biblioteca sarmentina por Ruelle, Fuchs, Dodoens, Mattias Lobel, Caspar Bahuin, Clusius..., todos ellos precursores de la descripción y clasificación de las plantas; la obra de Emanuel Sweert, Florilegium Amplissimum et Selectissimum (Fráncfort, 1612), ilustraciones botánicas de las nuevas plantas que se estaban introduciendo en Europa a principios de siglo; la Anatomia plantarum y las Opera posthuma (Londres, 1686), de Marcello Malpighi, que suponía analogías en el orden natural entre los tres reinos; el Methodus Plantarum Nova, de John Ray (Londres, 1682), considerado por Linneo como el fundador de la Sistemática; y la obra de Jacques Barrelier, Plantae per Galliam, Hispaniam et Italiam observatae (París, 1714).

Muchas de las obras mencionadas hasta el momento fueron consultadas por nuestro benedictino, citándolas en sus trabajos dedicados a la Historia Natural, en especial en el Catalogo de voces de diferentes vegetables, donde predominan las referencias a Dodoens, Plinio, Bauhin y Tournefort. Cabe destacar la obra de Tournefort, cuya clasificación estructuraba el orden de las plantas a partir de las características descriptivas. En los viajes de nuestro autor a Galicia le acompañan la obra de Bauhin, Historia plantarum universalis (Yverdon, 1651), y, en el de 1754, también la de Tournefort, Institutiones rei herbariae (Lyon, 1719), que le servía para herborizar. En sus Pensamientos critico-botanicos explica cómo recogía las plantas registradas en el Catalogo de voces de diferentes vegetables y la utilidad de las obras de Tournefort y Bahuin:

\footnotetext{
"Traje á Madrid 3 Baules llenos de vegetables; y de mixtos de la Historia Natural. El methodo que tube para los vegetables, ès el siguiente. Asi que yo veìa una planta que no era de las comunissimas, ó con flor, ó con fruto, ó con uno, y otra, la arrancaba por mi mismo, enseñabalà á este, ó á esta, preguntaba el nombre gallego, si le tenia; y que virtudes la atribuian los del Pais. Apuntabalo todo en mis Quadernos con una ligera descripcion, incluìa en un sobrescrito, la flor, semilla, y una hoja; y con este sobrescrito, a tantos de tal mes y año: en tal sitio individual, encontré esta planta, con este nombre; y con el numero, para reclamo en el q1. hable de ella en mis Quadernos.

Si era Planta, que no tenía nombre Gallego; recurrìa, para saber que nombre tenia en los Libros, a Tournefort, que llevè conmigo... Ó si queria saber virtudes etc. recurria à los tres tomos corpulentos de Juan Bauhino... Los Bauhinos, que tenia eran prestados de un Boticario, que no los usaba mucho" 53 .
}

\footnotetext{
${ }^{53}$ SARMIENTO, Martín: Pensamientos critico-botanicos..., fols. 371v.-372r.
} 
Junto a estos autores en el catálogo están las primeras obras renacentistas que describen los vegetales de América, como las de Nicolás Monardes y José Acosta, de gran importancia, especialmente el primero, por la introducción de nuevos remedios terapéuticos. Ambos aparecen citados por Sarmiento en sus trabajos Sobre los Vegetables Kali, Sosay Barrilla ${ }^{54}$ y De la Grama oloròsa en Gallego Lesta ${ }^{55}$.

Linneo, con quien parece que Sarmiento mantuvo correspondencia recibiendo en su celda a algunos de sus discípulos ${ }^{56}$, es el autor de Historia Natural de quien posee un mayor número de títulos:

"Mucho me hubiera alegrado tener el año de 755 en Pontevedra, las obras de Carlos Linnèo Sueco; a lo menos su tomo, genera plantarum. Vive hoy [1762] ese Autor, y es uno de los mas celebrados Botanistas del Mundo. Inbentò el Sistema de Conceder, Sexo a todo Vegetables... al presente tengo bastantes tomos del dicho Linneo; y estoy hecho cargo de todo su Sistema" 57 .

Estas obras de Linneo que compró Sarmiento son las que aparecen en su catálogo, como la tercera edición de Fundamenta Botanica (Ámsterdam, 1741); dos ediciones de Genera Plantarum (Leiden, 1742 y París, 1743); dos tomos del Systema Naturae (Lipsia 1748 y 1749); la primera edición de las Species Plantarum (Estocolmo, 1753); la primera edición de la Flora Lapponica (Ámsterdam, 1737); una edición de la Flora Suecica (Leiden, 1745); la primera edición de la Critica Botanica (Leiden, 1737), con el prólogo del botánico Johannes Browallius; y la primera edición de Philosophica Botanica (Estocolmo, 1751).

Pero la adopción del método de Linneo no le hace despreciar a nuestro autor a Tournefort, y para ejemplificar la variedad de los sistemas de ordenación compara Sarmiento los vegetales y los libros, los botánicos y los bibliotecarios: nunca estarán de acuerdo con la ordenación de otro colega, y aun así "podrá uno comprender todo el libro, y leerle de verbo ad verbum" 58 .

En sus estanterías están también algunos precursores de Linneo, como Sébastien Vaillant, y botánicos de su círculo, como el médico Hermann Boerhaave y Johannes Burman.

Otra figura fundamental de la Historia Natural en el siglo XVIII, George Louis

\footnotetext{
54 SARMIENTO, Martín: Sobre los Vegetables Kali, Sosa y Barrilla, fol. 39r.

55 SARMIENTO, Martín: De la Grama oloròsa en Gallego Lesta, fol. 796r.

56 SARMIENTO, Martín: Fray Martín Sarmiento. Epistolario, p. 230.

57 SARMIENTO, Martín: Pensamientos critico-botanicos..., fols. 373r.-373v.

${ }^{58}$ SANTOS PUERTO, José: Martín Sarmiento. Ilustración, educación y utopía en la España del siglo XV III, A Coruña, Fundación Pedro Barrié de la Maza, 2002, p. 398.
} 
Leclerc, el conde de Buffon, figura también en la colección sarmentina. De la Histoire naturelle posee Sarmiento 7 volúmenes (París, 1750 y 1758) de los 44 que acabarían de publicarse en 1789, muchos años después de su muerte. En el catálogo se destaca la participación en esta obra del anatomista Daubenton. Aparecen, además, entre los libros de nuestro autor, obras de divulgación, como el Espectáculo de la Naturaleza de Noël Antoine Pluche, traducido por Esteban de Terreros (Madrid, 1753).

Por su valor tipográfico hay que destacar también las Plantae selectae (Núremberg, 1750-1773), de Christoph Trew, una de las principales obras botánicas del siglo XVIII de la que Sarmiento afirma tener láminas de 30 plantas. Esta obra es fruto de la colaboración entre su autor, el pintor Georg Dionysius Ehret y el grabador Johann Jakob Haid.

Los últimos libros de botánica española que parecen entrar en la biblioteca de nuestro benedictino son la Flora española (Madrid, 1762), de Quer ${ }^{59}$, que en la obra reconoce el papel jugado por "su íntimo amigo" Sarmiento en su viaje a Galicia en 1761; la Historia Naturaly Médica (Madrid, 1762), de Gaspar Casal, que se ocupa de los minerales y las plantas de Asturias; y los Principios de Botánica (Madrid, 1767), de Miguel Barnades, uno de los introductores del método de Linneo en España. Y, dentro del género de los catálogos de coleccionistas, el Catalogue Systématique et Raisonné des Curiosités de la Nature et de l'Art, de Pedro Franco Dávila, editado en París en 1767 y base de la oferta de la venta al rey Carlos III.

Aparecen también en este epígrafe del catálogo de la biblioteca de Sarmiento obras que recogían las aplicaciones botánicas en la Medicina, obras de químicos y médicos, como la Materia medica de Étienne-François de Geoffroy, de la cual posee nuestro autor dos tomos en edición latina (Venecia, 1742) y diez tomos en edición francesa (París, 1743 y 1750); y el Dictionnaire universel des drogues simples de Nicolas Lémery, en su tercera edición ampliada con láminas (París, 1748). Sarmiento cita este último libro en 1762, en su Papel Curioso Sobre la Planta Seyxêbra ${ }^{60}$.

Los libros agrícolas no faltan en la biblioteca sarmentina, “...Dios no había puesto a Adán á otro oficio que al de Agricultor" ${ }^{61}$, Sarmiento será agricultor en su celda, intercambiando semillas y recibiendo tierra y también semillas de Galicia ${ }^{62}$.

En su catálogo a los clásicos de la Botánica le siguen los clásicos de la agricultura

\footnotetext{
${ }^{59}$ Sarmiento escribe Dedicatoria para la Obra dela Flora Española de Dr D" Joseph Quèr (AGFCMS, CM, T.XI, 1760, fols. 2r.-6v.).

${ }^{60}$ SARMIENTO, Martín: Papel Curioso Sobre la Planta... Seyxêbra, fol. 541v.

${ }^{61}$ SARMIENTO, Martín: "Carta sobre la historia natural, escrita á un caballero, residente en Londres, (1764)", p. 414.

62 Un ejemplo de estos envíos lo podemos encontrar en la carta escrita por Sarmiento a su hermano Francisco Javier, en Madrid, el 15 de febrero de 1758, SARMIENTO, Martín: Fray Martín Sarmiento. Epistolario, pp. 94-96.
} 
romana: Catón, Varrón, Columela, Paladio, Eliano y Apicio con su Re culinaria (Ámsterdam, 1709), que junto a las recetas añadía propiedades de los alimentos. También en estos libros nuestro benedictino atiende a la lengua: "Galicia, mi Patria, es la Provincia que más voces Latinas conserva, y en especial en quanto toca á agricultura. Dígolo porque leí, por curiosidad, de verbo ad verbum, á Catón, Varrón, Columela, y Paladio" "63.

Otros de los títulos que figuran en el catálogo en relación a esta materia son: la Agricultura (Medina del Campo, 15..), de Gabriel Alonso Herrera, citada tantas veces por Sarmiento; la obra de Jean Liebault y Charles Étienne L'Agriculture, et Maison rustique (París, 1598); L'Économia del cittadino in villa (Bolonia, 1651), de Vincenzo Tanara, presentando una agricultura enfocada a la búsqueda del beneficio y no a la subsistencia, aunque no carente de sugerencias gastronómicas; y el Dictionnaire oeconomique de Noël Chomel (Lyon, 1712). Están presentes también los textos de Duhamel de Monceau, el Traité de la culture des terres (París, 1753), una adaptación de la obra de Tull que se convirtió en el más importante tratado agronómico francés del siglo XVIII, y el Traité de la conservation des grains (París, 1753-1755).

Sobre el uso de los alimentos, junto al libro de Apicio, tiene Sarmiento la obra de Bartolomeo Scappi Dell'arte del cucinare (Venecia, 1570), que también hacía observaciones sobre animales y plantas; De re cibaria (Lyon, 1560), de Jean Bruyérin-Champier, que reúne la historia de los alimentos conocidos, la cocina y la dietética; la obra de Ludovicus Nunnius sobre la incidencia de la comida en la salud partiendo de los hábitos alimenticios de los romanos, Diaeteticon, sive de re cibaria (Amberes, 1645); y el libro de Baldasar Pisanelli Trattato de la natura de cibi et de Bere (Venecia, 1584).

Sarmiento manifiesta que los animales existen en menor número que las plantas ${ }^{64} \mathrm{y}$, paralelamente, podemos decir que sus libros zoológicos están en menor número que los botánicos en su biblioteca. Aun así, no falta una colección importante de textos de Zoología, especialmente renacentistas. Además de la obra de Claudio Eliano, de la de Demetrio Pepagomenus o del comentario de Julius Caesar Scaliger a la obra de Aristóteles sobre los animales (base de los conocimientos medievales de Zoología que tienen nueva expresión en el libro también aquí presente de Edward Woton, De differentiis animalium (París, 1752), posee Sarmiento otros trabajos zoológicos del Renacimento, como la obra de Rondelet, los libros de Conrad Gesner y Ulisse Aldrovandi (ambos padres de la entomología), la descripción del

\footnotetext{
${ }^{63}$ SARMIENTO, Martín: Memorias para la historia de la poesia, y poetas españoles, Madrid, Joaquín Ibarra, 1775, p. 144.

${ }^{64}$ SARMIENTO, Martín: Digresion sobre la Educación de la Juventud Española; desterrando el estudiar de memoria, y a la letra: y el Castigo: que son los dos Protectores de la ignorancia y del odio à las Letras, MP, CM, T.XVII, 17641765, fol. 166r.
} 
elefante de Gilles y los grabados de conchas de Fabio Columna. De Aldrovandi debió comprar Sarmiento sus obras en diferentes momentos pues los siete volúmenes de libros de este autor aparecen dispersos en esta parte del catálogo y es uno de los autores citados por él en diversas ocasiones. También posee Sarmiento De bumani corporis fabrica de Vesalio (Lyon, 1552), obra antecesora de los estudios de anatomía animal comparada, que se había continuado con la obra cumbre de Pierre Bellon du Mans, la Histoire de la Nature des Oyseaux, y que anota nuestro autor en su primera edición de 1555 (París). Sin duda la Zoología tiene su gran representante en el siglo XVII en las obras del médico John Jonston, a quien correspondió iniciar las bases de la taxonomía moderna y cuyas obras también constan en el catálogo de nuestro benedictino.

En menor número aún están los tratados minerológicos, aunque tiene Sarmiento algunas de las obras que marcan el inicio de la mineralogía científica: De re metallica (Basilea, 1556), de Georgius Agricola; el Arte de los metales (Madrid, 1729), de Álvaro Alonso Barba, un tratado del siglo XVII de metalurgia americana, editado junto a la historia de las minas en España de Alonso Carrillo; y la traducción del libro de Barba al francés por Lenglet Dufresnoy (París, 1751). Otros libros enlazan más con las tradiciones greco-romanas y medievales, como la Mineralogía de Bernard Cesius (Lyon, 1636), que incluye novedades sobre las virtudes de las piedras sacadas de la Cábala y de la Alquimia; la obra de Anselmo Boecio de Boot; la de Gaspar de Morales; así como el comentario a la obra de Plinio de Erasmus Stella. Algunos de los textos que aparecen aquí están relacionados con las aplicaciones prácticas de los minerales, tales como las médicas o las de tinturas.

En el catálogo también se registran algunos textos del inicio de la Paleontología, como la obra de Jacok Theodor Klein, Ordre naturel des oursins de mer et fossiles (París, 1754), y la de Louis Bourguet, Mémoires pour servir à l'bistoire naturelle des petrifications dans les quatre parties du monde (La Haya, 1742), uno de los primeros trabajos paleontológicos publicados en Francia.

Sarmiento aprovecha cualquier libro de cualquier materia que le pueda proporcionar información. Cuando Francisco de Paula Cousiño realiza sus apuntes para escribir una biografía de nuestro benedictino anota algunos de los libros que leyó "enteramente" Sarmiento, entre ellos, el del médico medieval Julián Gutiérrez de Toledo, Cura de la piedray dolor de la ijada, solo con fin de entresacar voces castellanas antiguas de vegetables (Toledo, 1498) ${ }^{65}$.

${ }^{65}$ COUSIÑO, Francisco de Paula: [Papeles de Don Francisco de Paula Cousiño sobre Fr. Martín Sarmiento], BUSC, 1748-1821, Ms. 599/3. De esta lectura, Sarmiento deja constancia en Cura de la Piedra, $y$ dolor de la Yjada, y colica rrenàl (s.a., MP, CM, T.II, fols. 296r.-300v.). 


\section{Leyendo para curar}

En cuanto a las obras de Medicina, la teoría y práctica médica aparecen a lo largo de la obra de Sarmiento, pero apenas han sido objeto de estudio ${ }^{66}$ y siguen sin serlo en los 20 años que han transcurrido desde entonces. Nuestro autor, en la línea que también representaba Feijoo, adoptará desde sus primeros escritos una posición crítica contra la Medicina que se estaba desarrollando en España, como ya señalamos al referirnos a su obra Martinus contra Martinum. Sarmiento propondrá la necesidad de la aplicación del método científico. Si hay un tema médico que interese al benedictino es la Terapéutica, considerando a la naturaleza la fuente del arte de sanar, y por tanto prestará una especial atención a la medicina popular y al poder curativo de las plantas. Por ello, no es de extrañar que las primeras obras situadas en el epígrafe Medicina. Cirugía y Quimica del catálogo sean ediciones de Hipócrates. La citada en primer lugar es la primera edición greco-latina de la obra completa hecha por el humanista alemán Anutius Foës (Fráncfort, 1595), que había expurgado el corpus hipocrático de las adendas que consideraba espurias, y que contiene la primera edición latina del autor, reeditada hasta principios del siglo XIX. Del mismo Foës consta en el catálogo el vocabulario de los términos griegos de Hipócrates Aeconomia Hippocratis alphabeti serie distincta (Fráncfort, 1588).

A las ediciones de Hipócrates sigue una relación de obras de autores médicos latinos, griegos, bizantinos y árabes, con frecuencia en ediciones del siglo XVI: Celso, Ecio, Alejandro de Tralles, Pablo de Egina, Areteo de Capadocia, Galeno, Avicena, Juan Mesué, Al-Farabi, Juan Actuario, Nicolás Myrepsos, etc. Con el esquema habitual de disposición de los libros en los epígrafes del catálogo, Sarmiento da paso a la medicina medieval con la obra de quien era una de las figuras más importantes en la materia de los siglos XII y XIII, Egidius Corboliensis (Gilles de Corbeil), cuyos poemas, De pulsibus y De urinis (Basilea, 1529), constituyeron auténticos libros de texto para los estudiantes de Medicina durante la Baja Edad Media. Otros autores medievales de la biblioteca son Matteo Silvatico, con su obra sobre la aplicación de las hierbas a la terapéutica Opus Pandectarum Medicinae (Venecia, 1507); Bernard de Gordon, con su tratado Lilium medicinae (Lyon, 1551) sobre la peste y otras enfermedades infecciosas; la Opera omnia (Lyon, 1532) de Arnau de Vilanova; y la Sevillana medicina de Juan de Aviñón, en su primera edición publicada por Nicolás Monardes en 1545

${ }^{66}$ GARCÍA GUERRA, Delfín: “La medicina en la obra del Padre Sarmiento”, pp. 348-352. 
(Sevilla), de la que nuestro benedictino indica en el catálogo que es rara.

De las obras de este período hay que destacar la Visita y Consejo de médicos de Estéfano de Sevilla, un manuscrito del siglo XV, "Rarísimo y acaso Códice único" "67. Sarmiento apenas ofrece datos sobre este códice en el catálogo, sin embargo, nos dejó un texto sobre el mismo con el título Sobre un Codice M.S. to en Castellano, de Medicina, su Autor Estephano, natural de Sevilla ${ }^{68}$. En este texto nuestro autor describe el manuscrito como de 37 pliegos de papel tosco, escrito en castellano, aunque con título en latín, con letra del tiempo de Juan II, rúbricas e iniciales en bermellón, y falto de folios al principio, medio y final. El manuscrito se encuentra en la actualidad en la Biblioteca Nacional, procedente de Pascual de Gayangos, con signatura Ms. 18052. No hay duda de que se trata de la copia que poseía Sarmiento, ya que en la hoja 1 tiene la anotación: "Folié este libro año de 1739. Fr. Martín Sarmiento, benedictino", constituyendo así una buena muestra de la dispersión de la biblioteca de nuestro autor, como de tantas otras, tras la desamortización.

De la medicina renacentista están en la colección sarmentina las obras de Luis Lobera de Ávila, con gran atención a la alimentación (Alcalá, 1542, 1544 y 1551); la Curationium Centuriae Septem (Barcelona, 1628), del portugués Amato Lusitano, una obra de casos clínicos; Practica Valesci de Tharanta que alias Philonium dicitur (Venecia, 1523), del también portugués Vasco de Taranta, un libro de práctica médica; y Observationum medicarum rariorum liber (Fráncfort, 1665), un compendio de casos patológicos que reunía observaciones de Johannes Schenck von Grafenberg (médico alemán considerado un pionero de la neurolingüística) y de otros médicos desde la antigüedad.

En el siglo XVI se produjo una considerable ampliación de los conocimientos botánicos en materia médica gracias a los viajes naturalistas, aquí representados por la obra de Próspero Alpino. Sarmiento incluye las obras De medicina Aegyptiorum y De Plantis Aegyptiorum entre los Libros de singulares asuntos en el Catalogo de libros curiosos y selectos ${ }^{69}$. Ambas obras están en su biblioteca (Venecia, 1591 y 1592), como también la del botánico polaco Anton Schneeberger Medicamentorum facile parabilium adversus omnis generis articulorum dolores enumeratio (Fráncfort, 1581). Entre los autores españoles cabe destacar a Olivia Sabuco y Antonio Gómez Pereira.

De los autores del siglo XVII encontramos los dos primeros tomos de la obra del

\footnotetext{
${ }^{67}$ SARMIENTO, Martín: Catalogo de los Autores, de quienes, yo Fr. Martin Sarmiento..., fol. 163r.

${ }^{68}$ SARMIENTO, Martín: Sobre un Codice M.S. to en Castellano, de Medicina, su Autor Estephano, natural de Sevilla, MP, CM, T.II, 1752, fols. 270r.-276r.

${ }^{69}$ SARMIENTO, Martín: Catalogo de algunos libros curiosos y selectos, para la libreria de algun particular, que desee comprar de tres á quatro mil tomos, Henrique Monteagudo (ed.), A Coruña, Toxosoutos, 2002, p. 168.
} 
químico Daniel Sennert con las primeras aportaciones a la teoría atómica (Lyon, 1650), aunque posteriormente adquirirá otro tomo con sus Epístolas (Lyon, 1666). Entre los variados fondos de este siglo, hay que destacar la obra de William Harvey que, aunque es más conocido por sus contribuciones al conocimiento de la circulación de la sangre, hizo aportaciones muy significativas a la embriología en Exercitationes de generatione animalium, cuya primera edición de 1651 (Ámsterdam) tiene Sarmiento. De Harvey recogería ideas el iatroquímico Thomas Willis, aunque también estudió el sistema nervioso, la anatomía descriptiva y la farmacología, y tuvo gran difusión en la medicina europea. Nuestro autor posee su Opera omnia editada en Venecia en 1720. A esta misma corriente pertenecía Johannes Baptista van Helmont y Giorgio Baglivi, ambos presentes en el catálogo con ediciones de sus Opera Omnia (Fráncfort, 1707 y Venecia, 1723 respectivamente). Del anatomista francés Raymond Vieussens, que se reclama deudor de Willis, tiene Sarmiento la primera edición de Neurographia universalis (Lyon, 1684), una obra sobre neuroanatomía. Y del médico inglés Richard Morton, estudioso de la tuberculosis y el primero que describe la anorexia, figura en esta colección una edición de la Opera medica (Lyon, 1718).

Entre los libros del siglo XVIII seguimos encontrando obras sobre estos temas: la circulación de la sangre (Jacob Keil, Leiden, 1730); las infecciones (Jacob Henriques de Castro Sarmento, Leiden, 1722); textos médicos sobre las enfermedades venéreas, tales como Aphrodisiacus, en la edición de Hermann Boerhaave (Leiden, 1728) y De morbis venereis, de Jean Astruc (Venecia, 1748); de parasitología (Nicholas Andry de Boisregard, París, 1741); de anatomía (Frederik Ruysch, Ámsterdam, 1701 y 1728); de terapéutica (Anton de Haen, París, 1761) y farmacología (Jean Baptiste Martinenq, París, 1748).

En cuanto a la bibliografía médica española, de autores de comienzos del siglo XVIII posee Sarmiento la obra Medicina sceptica (Madrid, 1722), del novator Martín Martínez, quien representa un renovado hipocratismo en la medicina española ${ }^{70}$. A este mismo autor atribuye Sarmiento la obra firmada por Francisco Perena Conclusiones breves y claras, theológico-médicolegales contra la Disertación Médico-Theológica que dio a la luz Diego Matheo Zapata (Madrid, 1733). Posee también nuestro benedictino obras de Francisco Suárez de Ribera (quien polemizaría con Feijoo), Francisco Alonso Esteban y Lecha y Vicente Lardizábal.

Junto a estas y como él recomendaba tiene nuestro autor una historia de la Medicina, Histoire de la médicine depuis de Galien (Leiden, 1727), de John Freind; una bibliografía de autores médicos, la Bibliotheca scriptorum medicorum (Ginebra, 1731), del mismo autor; y un vocabulario

${ }^{70}$ LÓPEZ PIÑEIRO, José María: "La mentalidad antisistemática en la medicina española del siglo XVIII. La influencia de la Alte Wiener Schule", en Medicina moderna y sociedad española, Valencia, Cátedra e Instituto de Historia de la Medicina, 1976, pp. 191-214. 
y una bibliografía de anatomía, Dictionnaire anatomique, suivi d'une Bibliothèque anatomique et physiologique (París, 1753), de Pierre Tarin. Sarmiento adquirió libros de Medicina hasta el final de su vida, ya que entre ellos está el Tratado completo de calenturas de José Luis Pereira editado en Madrid en 1768.

\section{Conclusiones}

El interés de Sarmiento por la Historia Natural, a la que considera la base de todas las ciencias, y en especial por la Botánica, así como por la Medicina, es notorio en sus escritos. Nuestro autor realiza numerosos trabajos sobre diferentes asuntos de Historia Natural en los que deja constancia, además, del método para escribir y estudiar dicha materia y su aplicación a la Medicina. En este sentido, Sarmiento prima la observación y la experiencia: los datos empíricos proporcionados por la práctica clínica deben constituir el arte de curar. Defiende también la necesidad del conocimiento de la Química como uno de los fundamentos de la naturaleza y cree y apoya la fuerza sanadora de esta, una corriente terapéutica que aboga por la utilización de los remedios naturales aprovechando las virtudes medicinales de las plantas y el conocimiento popular.

Dentro del conjunto de las colecciones científicas de la biblioteca de Sarmiento ninguna colección es tan destacable como la Historia Natural, Botánica y Agricultura. En ella confrontó muchas de sus identificaciones y descripciones de plantas y sus usos, aplicando sus conocimientos botánicos a la agricultura, la farmacología, la química, la dietética, la etimología y el método de estudios. Recordemos que defiende un modelo pedagógico en el que el aprendizaje se fundamenta en la tradición (apoyado en el contacto con los elementos de la naturaleza) y la lengua materna.

Compara nuestro autor las plantas con los libros, ya que en ambos, los esfuerzos de la época se sustentaban en la descripción y la clasificación. Ninguna materia representa tan bien en la biblioteca sarmentina la ciencia ilustrada, una ciencia que busca el bienestar y persigue su aplicabilidad. Su afición por la Medicina compartía iguales objetivos, como muestra su colección de obras médicas, cuyos libros, siempre atentos a la terapéutica y la farmacología, trataban también las enfermedades más frecuentes en la época (infecciones, enfermedades venéreas, parasitología).

Sarmiento señala como métodos de investigación (especialmente para su dedicación a la Historia Natural) tanto el trabajo de campo como el de gabinete, a los que acompaña de pequeñas ilustraciones y de las citas bibliográficas de las fuentes en las que se basa. 
Precisamente de sus fuentes librarias extrajo las abundantes citas que se insertan en sus textos y que son una muestra de su cultura libresca, en buena parte obtenida de su propia biblioteca. Criticado con frecuencia por su erudición bibliográfica, que entorpece a veces la lectura de sus textos, los escritos de nuestro autor suelen nacer con conocimiento de causa, bien justificando su posición en otros autores, bien criticándolos. Pero ello no impide que en alguna de sus cartas al duque de Medina Sidonia afirme que sus conocimientos los debe a sus sentidos exteriores y a la experiencia, y que "Más me deleita una hoja de un vegetable, que una hoja de un libro en folio" ${ }^{, 71}$.

\section{Siglas}

AGFCMS. Archivo General de la Fundación Casa Medina Sidonia

BUSC. Biblioteca Universitaria de Santiago de Compostela

CM. Colección Medina Sidonia

MP. Museo de Pontevedra

BNE. Biblioteca Nacional de España

RAH. Real Academia de la Historia

${ }^{71}$ SARMIENTO, Martín: Cartas al Duque de Medinasidonia, 1747-1770, p. 175. 